\title{
Impact of Out-of-Pocket Pharmacy Costs on Branded Medication Adherence Among Patients with Type 2 Diabetes
}

\author{
Wendy S. Bibeau, PhD, MEd; Haoda Fu, PhD; April D. Taylor, MSN, CNS, BC-ADM;
} and Anita Y.M. Kwan, MSc

\begin{abstract}
BACKGROUND: Medication adherence is pivotal for the successful treatment of diabetes. However, medication adherence remains a major concern, as nonadherence is associated with poor health outcomes. Studies have indicated that increasing patients' share of medication costs significantly reduces adherence. Little is known about a potential out-of-pocket (00P) cost threshold where substantial reduction in adherence may occur.
\end{abstract}

OBJECTIVE: To examine the impact of diabetes 00P pharmacy costs on antihyperglycemic medication adherence and identify the potential threshold at which significant reduction in adherence may occur among patients with type 2 diabetes mellitus (T2DM).

METHODS: This was an observational, retrospective cohort study using longitudinal U.S. pharmacy and medical claims data from the IMS Health Medical Claims (Dx) database. Patients with T2DM who initiated therapy with a branded antihyperglycemic medication during the index period (January 1, 2011, to December 31, 2011) and had 3 years of follow-up data were included. The primary outcome was adherence to antihyperglycemic medications, measured as the number of days covered. Propensity scores were calculated using baseline sociodemographic and clinical characteristics to control for potential confounding factors. Four strata were created based on mean propensity scores. Across each stratum, patients were assigned to 5 diabetes 00P pharmacy (including generics) cost levels: $\$ 0-\$ 10, \$ 11-\$ 40, \$ 41-\$ 50, \$ 51-\$ 75$, and $>\$ 75$. Multivariate regression models were used to estimate association of diabetes 00P pharmacy costs and adherence for each stratum. Sensitivity analyses were conducted to assess the impact of total 00P pharmacy costs and index drug category OOP costs on adherence.

RESULTS: A total of 15,416 patients were assessed. Across each stratum in the diabetes 00P pharmacy cost analysis group, mean patient age ranged from 52.3 to 56.1 years, mean number of antihyperglycemic medication classes ranged from 1.5 to 3.2 , and mean household income ranged from $\$ 60,763$ to $\$ 79,373$. Most patients used a commercial plan (55\%-85\%). The propensity-stratified multivariate regression model revealed an overall negative relationship between diabetes 00P pharmacy costs and adherence across several 00P cost levels. Diabetes 00P pharmacy cost level $\$ 51-\$ 75$ appeared as the threshold at which adherence reduced significantly (77-78 fewer days of coverage over 3 years of follow-up; $P<0.05$ ) when compared with the lowest 00P costs $(\$ 0-\$ 10)$ across all strata. Adherence reduced further $(99-145$ fewer days of coverage; $P<0.0001)$ for the higher diabetes 00P pharmacy cost levels $(>\$ 75)$ when compared with the lowest 00P cost levels. Sensitivity analyses with total 00P pharmacy costs and index drug category 00P costs revealed negative association with adherence across all strata.

CONCLUSIONS: Diabetes OOP pharmacy cost was negatively associated with patient adherence, and a potential 00P cost threshold (\$51-\$75) was identified at which adherence reduced significantly. The study findings may be beneficial in informing the design of health care plans to achieve optimal adherence and improve disease management in patients with T2DM.

J Manag Care Spec Pharm. 2016;22(11):1338-47

Copyright $\odot 2016$, Academy of Managed Care Pharmacy. All rights reserved.

\section{What is already known about this subject}

Adherence to therapy is a critical component in the successful management of diabetes. However, studies indicate that less than $50 \%$ of patients achieve glycemic goals, which may be attributable to low medication adherence in patients with type 2 diabetes mellitus (TD2M).

Medication nonadherence has been reported to lead to poor clinical outcomes, high resource utilization, and increased costs in patients with diabetes. Out-of-pocket (OOP) costs have been shown to be negatively associated with medication adherence in patients with diabetes.

New strategies that shift costs to the patient in order to drive patient awareness about cost of care are, in part, responsible for the increase in patient OOP costs. However, studies suggest that switching to a health care plan that includes modest drug copays may not reduce medication use or lead to reduced adherence for chronic diseases.

\section{What this study adds}

This study provides contemporary evidence that antihyperglycemic medication adherence differs by OOP cost levels in patients with T2DM.

The study findings indicate that there is a threshold at which OOP costs become a substantial barrier to attaining antihyperglycemic medication adherence. Diabetes OOP pharmacy cost level \$51-\$75 was identified as the potential threshold at which significant reduction in adherence was observed. Sensitivity analysis revealed a higher threshold (OOP cost levels \$91-\$150) for total OOP pharmacy costs.

These results can be used to inform plan designs and tier structure to ensure copays do not negatively and substantially affect medication adherence and thereby help to attain optimal adherence and reduce overall health care expenditures in patients with diabetes.

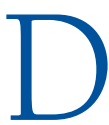
iabetes mellitus is a complex, multifactorial disease that affects approximately 29 million people in the United States. ${ }^{1}$ Diabetes and associated complications are expected to be the seventh leading cause of death by $2030 .^{2}$ Type 2 diabetes mellitus (T2DM) is the most common type and accounts for 90\%-95\% of all diagnosed cases. ${ }^{1}$ Lifestyle changes are recommended as the first-line therapy for patients 
with T2DM, and oral antihyperglycemic medications, with or without insulin, are used if adequate glycemic control is not achieved or maintained by lifestyle changes. ${ }^{3}$ A critical component in the successful management of diabetes is adherence to therapy. ${ }^{4}$ However, studies indicate that less than $50 \%$ of patients achieve glycemic goals, which may be attributable, in part, to low adherence to therapies. ${ }^{5}$

Medication adherence is a major concern to providers, health care systems, and payers due to the increasing evidence that medication nonadherence is pervasive ${ }^{6}$ and associated with adverse health outcomes and high resource utilization (e.g., hospitalizations, emergency department [ED] visits, etc.) and costs. ${ }^{7}$ Medication nonadherence is responsible for substantial medication-related hospital admissions, ranging from $33 \%$ to $69 \%,{ }^{8}$ and the suboptimal rates of medication adherence $^{9}$ are estimated to cost the U.S. health care system between $\$ 100$ billion and $\$ 300$ billion annually. ${ }^{10}$ The medical cost burden of the least-adherent patients with T2DM $(\$ 21,421)$ is nearly 3 times higher than the most-adherent patients with T2DM $(\$ 7,692) .{ }^{11}$

Conversely, evidence suggests that individuals with better medication adherence have better health outcomes and lower rates of health care resource utilization. It has been estimated that improved adherence to antihyperglycemic medications could avoid 699,000 ED visits and 341,000 hospitalizations annually. ${ }^{12}$ Also, it is estimated that every additional dollar spent on adhering to a prescribed medication would reduce total health care costs by $\$ 6.7$ in patients with diabetes ${ }^{13}$ and improved adherence could potentially lead to annual health care savings of up to $\$ 8.3$ billion in the United States. ${ }^{12}$

With continual increases in health care spending, ${ }^{14}$ strategies are being used to curb the overuse of unnecessary medications and shift costs to the patient, such as increase in patient copayments and coinsurance, increase in formulary listing restrictions, and mandatory substitution of branded products with less expensive generics. ${ }^{15}$ A 2015 annual census of U.S. health insurance companies has shown that enrollment in health savings account/high-deductible health plans has almost doubled in the last 5 years. ${ }^{16}$ These strategies are partly the reason for the increase in patient OOP costs, which increased by $1.3 \%$ to $\$ 329.8$ billion in 2014 or $11 \%$ of total national health expenditures in 2014. ${ }^{14}$ Unfortunately, these strategies to reduce medication use may be counterproductive, as studies have indicated that increasing patient share of medication costs is significantly associated with a decrease in adherence. $^{17}$

However, a study has reported that switching to a health care plan that includes modest drug copays may not reduce medication use or lead to reduced adherence for chronic diseases. ${ }^{18}$ Therefore, it is essential for managed care decision makers to identify the point at which additional OOP costs adversely affect medication adherence. ${ }^{15}$ Although a few studies have assessed the impact of patient copay levels on adherence in patients with $\mathrm{T} 2 \mathrm{DM},{ }^{19-21}$ the threshold of OOP pharmacy costs that leads to significant reduction in adherence remains unclear. Also, the increasing trend of enrollment in high-deductible health plans in recent years indicates a need for ongoing research on the association of OOP costs and adherence.

The objective of this study was to examine the impact of diabetes OOP pharmacy costs on adherence and identify the threshold of diabetes OOP pharmacy costs at which copays become a substantial barrier to attaining antihyperglycemic medication adherence, regardless of income and sociodemographic and clinical characteristics.

\section{Methods}

\section{Data Source}

This study used longitudinal pharmacy and medical claims data from the IMS/Amundsen database. The IMS Health Medical Claims (Dx) database is a longitudinal dataset that captures information about patients' medical diagnoses, procedures, laboratory investigations, and other related medical claims activity from full-time office-based physicians across the United States. ${ }^{22}$ This dataset was used to identify other diagnosed comorbidities for the cohort of patients with diabetes and subsequently assign the appropriate number of points for the chronic disease score (CDS) ${ }^{23}$ and adapted diabetes complications severity index (aDCSI). ${ }^{24}$

In addition, data from the formulary impact analyzer (FIA) were used to identify OOP costs for each claim. The FIA is a transactional claim dataset derived from the interaction and information passing between pharmacy outlets and payer adjudication processes. The FIA is sourced from retail and mailorder pharmacies and is a nonprojected dataset (i.e., data are not projected up to estimate the total market, thereby allowing patient longitudinal analyses to be conducted) representing approximately $50 \%$ of claim volume for the diabetes market. Each claim was mapped to a de-identified patient in the IMS system using a proprietary and patented approach, enabling these FIA patients to be linked to the IMS system. Finally, the claims data were cross-matched with sociodemographic variables through partnership with Experian, the global information services company, and the impact of these variables on the outcomes of interest was evaluated.

\section{Study Design}

This study was an observational, retrospective cohort analysis of patients with T2DM aged 18-85 years who initiated 1 or more branded antihyperglycemic products within selected Uniform System of Classification categories (Appendix A, available in online article) during the index period (January 1, 2011, to December 31, 2011). Patients should have had (a) at least 2 claims with a T2DM diagnosis (International Classification of Diseases, Ninth Revision, Clinical Modification [ICD-9-CM] 250.x0, 
250.x2) on 2 dates during the pre-index period ( 6 months before the index date), (b) $\geq 6$ months' continuous eligibility in the pre-index period, and (c) $\geq 12$ months' continuous eligibility during the post-index period (3-year follow-up period). Patients with claims for a type 1 diabetes mellitus (ICD-9-CM 250.x1, 250.x3) or gestational diabetes (ICD-9-CM 648.8x) diagnosis during the pre-index period were excluded.

\section{Measures}

Demographic characteristics were summarized for the target population, including age and sex at index period, and income, level of education, geographic location, marital status, and insurance plan type (commercial, Medicare Part D) at index and follow-up periods. Clinical variables such as CDS (score ranging from 0 to 36 , with higher scores indicating poorer outcomes), ${ }^{23}$ aDCSI (score ranging from 0 to 13 , with higher scores indicating increased risk of complications), ${ }^{24}$ and targeted comorbidities of interest were captured at index period (Appendix B, available in online article).

The primary outcome measure was antihyperglycemic medication adherence, which was measured by the number of days covered during the follow-up period from January 1, 2012, to December 31, 2014. The number of days covered was defined as the number of days in the follow-up period during which the index medication was determined to be in hand based on the pharmacy claim fill date plus days supply. The follow-up period was 3 years for all patients.

The first-year mode copay was used to determine patient OOP costs for each drug category. Diabetes OOP pharmacy costs were calculated by adding all first-year mode copay amounts across all diabetes medications including generics. On the basis of the data retrieved and the most logical or frequently observed cut points, patients were assigned to 5 diabetes OOP pharmacy cost levels based on monthly OOP costs: $\$ 0-\$ 10$, $\$ 11-\$ 40, \$ 41-\$ 50, \$ 51-\$ 75$, and $>\$ 75$. Patients starting therapy in multiple index drug categories were assigned to cost cohorts in all the drug categories in which they initiated therapy during the index period, but the overall OOP costs were calculated at the patient level. The impact of diabetes OOP pharmacy costs on adherence was measured for patients for the OOP cost levels identified. For all patients, adherence as a function of diabetes OOP pharmacy costs was observed at the patient level, and patients were tracked over the follow-up period to determine the number of days covered.

\section{Statistical Analysis}

The association of diabetes OOP pharmacy costs and adherence was assessed using a propensity-stratified combined impact model. A logistic regression analysis was conducted wherein the dependent variable was OOP medication cost $>\$ 35$; the dependent variable, OOP medication cost $>\$ 35$, was identified based on a generally linear trend with a commonly observed point of inflection of about $\$ 35 .{ }^{25}$ Confounders were selected based on Pearson's correlation, and those with the highest coefficients were included. Confounders included age, income, number of diabetes medication classes, total number of diabetes medications, CDS, number of all medication classes, total number of all medications, marital status, and type of insurance.

Propensity scores were calculated to estimate the probability of OOP medication costs $>\$ 35$ using baseline sociodemographic and clinical characteristics. To minimize the impact of confounding variables, patients were divided into 4 strata based on Tukey's Five Number Summary. Matching was not done, and propensity score stratification was used for adjustment in the analysis. A multivariate basic regression model was conducted to estimate the association of diabetes OOP pharmacy costs and antihyperglycemic medication adherence for each stratum. Diabetes OOP pharmacy costs and the stratifications resulting from the propensity stratification were the independent variables, whereas total number of days covered for diabetes medications during the 3-year follow-up was the dependent variable in the multivariate analysis.

Variables missing on more than $10 \%$ of the claims transactions were not included in the analyses. However, if a variable was considered crucial to an analysis and the volume became an issue per the rule above, claims with populated fields were included in the analysis.

\section{Sensitivity Analysis}

Patients with diabetes are at a high risk of coexisting medical conditions and may require multiple medications to manage not only hyperglycemia but also associated comorbidities, such as dyslipidemia, hypertension, and depression. ${ }^{26}$ Therefore, a sensitivity analysis was conducted to assess the impact of total OOP pharmacy costs on adherence. The total OOP pharmacy costs were calculated by adding first-year mode copay amounts across all therapy area medications, including generics. Patients were assigned to 6 total OOP pharmacy cost cohorts based on monthly OOP costs: $\$ 0-\$ 30, \$ 31-\$ 60, \$ 61-\$ 90, \$ 91-\$ 150$, $\$ 151-\$ 200$, and $>\$ 200$. In addition, the association of the index diabetes medication (only 1 antihyperglycemic medication) OOP costs and adherence was also assessed across several monthly OOP cost levels (\$0-\$10, $\$ 11-\$ 40, \$ 41-\$ 50, \$ 51-\$ 75$, and $>\$ 75)$ in the sensitivity analysis to account for the effect of patients' characteristics at index period.

\section{Results}

At the beginning of the study, more than 6.5 million patients with diabetes were identified. After applying the inclusion and exclusion criteria, the majority of the patients were excluded because they had not initiated an index drug category treatment during the index period. On average, the index drug categories selected were more expensive than other agents such 
Diabetes patients identified from the database in 2011 $\mathrm{N}=6,581,414$
Excluded:

- Patients without branded index drug therapy initiation in 2011 , $n=6,000,000$

- Patients without look-forward eligibility, $n=50,956$

- Patients without medical claims eligibility, $n=195,781$

- Patients without T2DM diagnosis, $n=87,979$

- Patients without reliable copay information, $n=120,372$

- Patients wihout a clear copay mode, $n=9,351$

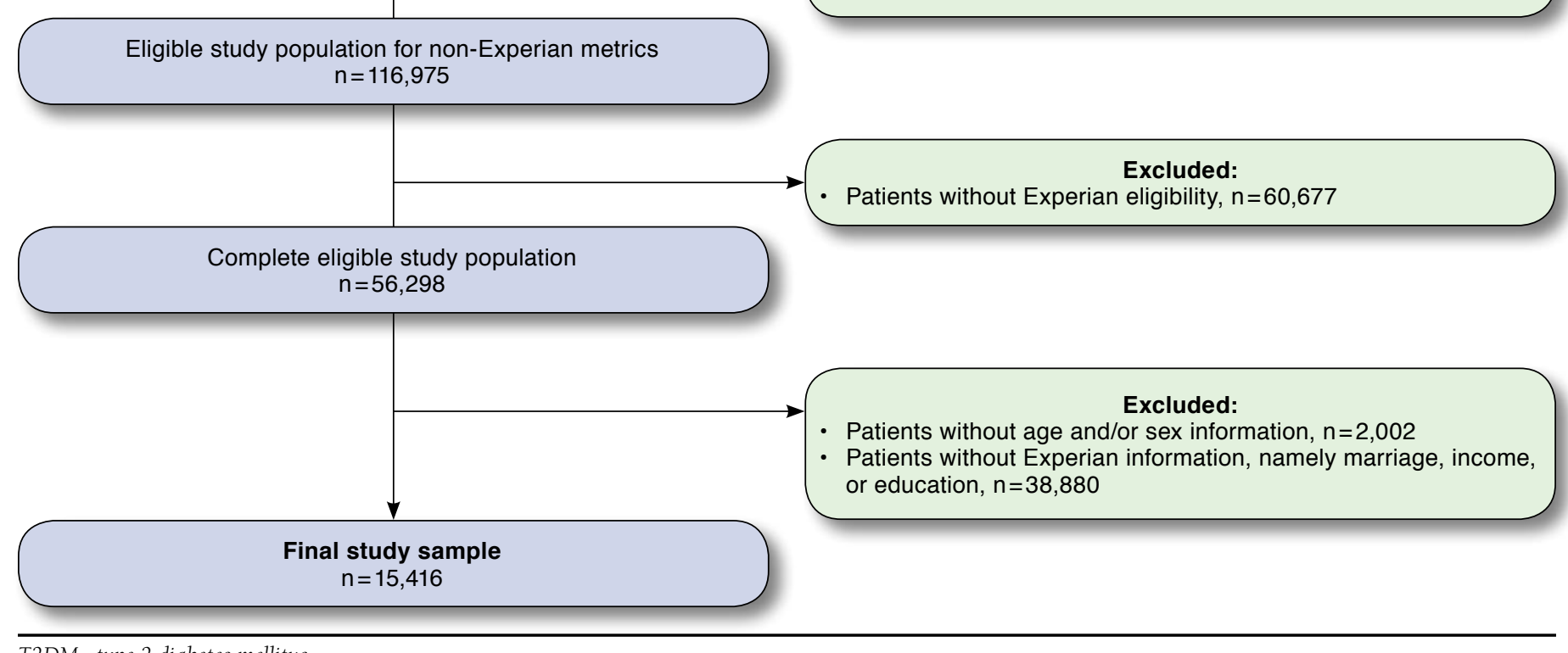

T2DM = type 2 diabetes mellitus.

as metformin and sulfonylurea that were not included as index drug categories, but patients had different insurance coverages that allowed some patients to fill the selected agents at little to no cost, thus allowing a variety of costs to be included in the assessment of adherence. Approximately 116,975 patients with therapy initiation in 2011 and pharmacy claims data for the entire 3-year study period were eligible for the study. Considering patients whose cost information and socioeconomic data were available, the final sample size for each model was 15,416 patients (Figure 1).

\section{Baseline Characteristics}

The categorization of the 4 strata based on the probability of having a copay of $>\$ 35$ is outlined in Table 1 . Stratum 1 had the lowest probability of high OOP costs, whereas stratum 4 had the highest probability of high OOP costs.

At the index period, although significant differences were observed for some covariates, overall the covariates were balanced across the 2 OOP cost groups $(>\$ 35$ and $\leq \$ 35)$. The

\begin{tabular}{l|c|c|c|c}
\hline \multirow{2}{*}{ TABLE 1 } & $\begin{array}{l}\text { Copay Stratification Based on the } \\
\text { Probability of OOP Medication } \\
\text { Costs }>\$ 35\end{array}$ \\
\cline { 3 - 5 } & & \multicolumn{3}{|c}{ Propensity Scores (\%) } \\
\cline { 3 - 5 } Stratum & $\mathrm{N}$ & Average & Minimum & Maximum \\
\hline 1 & 3,854 & 3.0 & 0.1 & 5.4 \\
\hline 2 & 3,854 & 7.8 & 5.4 & 10.1 \\
\hline 3 & 3,854 & 13.5 & 10.1 & 18.1 \\
\hline 4 & 3,854 & 29.5 & 18.1 & 90.0 \\
\hline OOP= out-of-pocket.
\end{tabular}

number of diabetes medications and diabetes therapy classes used at index period were similar across all 4 strata (Table 2A). Across each stratum, the mean patient age ranged from 46.6 to 61.6 years, mean CDS ranged from 5.4 to 7.0, mean number of diabetes medication classes ranged from 1.9 to 2.4 , and mean household income ranged from $\$ 62,893$ to $\$ 77,028$. Most patients were married (mean range, 62\%-98\%) and some used 


\begin{tabular}{|c|c|c|c|c|}
\hline Confounders & $\begin{array}{c}\text { Stratum } 1 \\
\mathrm{~N}=3,854 \\
\end{array}$ & $\begin{array}{l}\text { Stratum } 2 \\
\mathrm{~N}=3,854\end{array}$ & $\begin{array}{l}\text { Stratum } 3 \\
\mathrm{~N}=3,854\end{array}$ & $\begin{array}{l}\text { Stratum } 4 \\
\mathrm{~N}=3,854\end{array}$ \\
\hline \multicolumn{5}{|l|}{ A. Characteristics at Index Period } \\
\hline Age (years), mean \pm SD & $46.6 \pm 15.4^{\mathrm{a}}$ & $54.1 \pm 12.1$ & $57.3 \pm 12.2^{b}$ & $61.6 \pm 13.0$ \\
\hline Income, $\$$, mean $\pm S D$ & $64,377 \pm 48,174$ & $75,941 \pm 47,609$ & $77,028 \pm 48,897$ & $62,893 \pm 45,947^{\mathrm{a}}$ \\
\hline Diabetes medication class, ${ }^{\mathrm{c}}$ mean $\pm \mathrm{SD}$ & $2.4 \pm 1.1^{\mathrm{a}}$ & $2.2 \pm 0.9$ & $1.9 \pm 0.9$ & $1.9 \pm 0.9^{\mathrm{a}}$ \\
\hline Diabetes therapies, mean \pm SD & $2.8 \pm 1.1$ & $2.5 \pm 1.0^{\mathrm{a}}$ & $2.1 \pm 1.0$ & $2.1 \pm 1.0^{\mathrm{a}}$ \\
\hline $\mathrm{CDS}$, mean $\pm \mathrm{SD}$ & $7.0 \pm 3.2^{\mathrm{a}}$ & $6.3 \pm 2.9^{a}$ & $5.4 \pm 2.5$ & $6.2 \pm 3.0^{\mathrm{a}}$ \\
\hline Total medication class, ${ }^{\mathrm{d}}$ mean $\pm \mathrm{SD}$ & $9.1 \pm 3.9^{\mathrm{a}}$ & $7.6 \pm 3.4^{\mathrm{a}}$ & $5.7 \pm 3.0$ & $6.7 \pm 3.8^{\mathrm{a}}$ \\
\hline Total therapies, mean \pm SD & $9.6 \pm 4.0^{\mathrm{a}}$ & $8.1 \pm 3.4^{\mathrm{a}}$ & $6.2 \pm 3.1$ & $7.1 \pm 3.9^{\mathrm{a}}$ \\
\hline Married, \% & 62 & 96 & 95 & 98 \\
\hline Commercial, \% & 72 & 98 & 94 & $30^{\mathrm{a}}$ \\
\hline Part D, \% & 0 & $2^{a}$ & $5^{\mathrm{a}}$ & 60 \\
\hline Medicaid, \% & 28 & 0 & 0 & 0 \\
\hline \multicolumn{5}{|c|}{ B. Characteristics of the Diabetes OOP Pharmacy Costs Group } \\
\hline Age (years), mean $\pm \mathrm{SD}$ & $52.3 \pm 15.2$ & $55.6 \pm 15.2^{\mathrm{b}}$ & $56.1 \pm 14.0$ & $55.5 \pm 12.4$ \\
\hline Income, $\$$, mean $\pm S D$ & $60,763 \pm 43,577$ & $67,787 \pm 46,504 \mathrm{~b}$ & $72,312 \pm 48,630^{b}$ & $79,373 \pm 51,444$ \\
\hline Diabetes medication class, ${ }^{\mathrm{c}}$ mean $\pm \mathrm{SD}$ & $1.5 \pm 0.8^{\mathrm{a}}$ & $1.6 \pm 0.6$ & $2.2 \pm 0.5$ & $3.2 \pm 0.7$ \\
\hline Diabetes therapies, mean \pm SD & $1.8 \pm 0.9^{\mathrm{a}}$ & $1.8 \pm 0.8$ & $2.4 \pm 0.7$ & $3.4 \pm 0.9$ \\
\hline $\mathrm{CDS}$, mean $\pm \mathrm{SD}$ & $6.5 \pm 3.2^{\mathrm{a}}$ & $6.0 \pm 3.0$ & $6.3 \pm 2.9^{b}$ & $6.1 \pm 2.7^{\mathrm{b}}$ \\
\hline Total medication class, ${ }^{\mathrm{d}}$ mean \pm SD & $6.8 \pm 4.3^{a}$ & $6.5 \pm 3.5^{b}$ & $7.5 \pm 3.5^{a}$ & $8.3 \pm 3.5^{a}$ \\
\hline Total therapies, mean \pm SD & $7.4 \pm 4.3^{\mathrm{a}}$ & $6.9 \pm 3.6$ & $7.9 \pm 3.6^{\mathrm{a}}$ & $8.8 \pm 3.6^{\mathrm{a}}$ \\
\hline Married, \% & 76 & 89 & 92 & 94 \\
\hline Commercial, \% & 55 & $76^{\mathrm{b}}$ & $78^{\mathrm{a}}$ & 85 \\
\hline Part D, \% & 17 & 23 & 18 & $9^{a}$ \\
\hline Medicaid, \% & 28 & 0 & 0 & 0 \\
\hline \multicolumn{5}{|c|}{$\begin{array}{l}\text { Note: Patients were divided into } 4 \text { strata based on Tukey's Five Number Summary to minimize the impact of confounding variables. P values indicate comparison } \\
\text { OOP cost group versus }>\$ 35 \text { OOP cost group. } \\
\text { a P }<0.0001 . \\
{ }^{2} P<0.05 \text {. } \\
{ }^{c D i a b e t e s ~ m e d i c a t i o n ~ c l a s s ~ i s ~ d e f i n e d ~ a s ~ t h e ~ n u m b e r ~ o f ~ d i a b e t e s ~ d r u g ~ c a t e g o r i e s ~(i n c l u d i n g ~ i n j e c t a b l e s) ~ u s e d ~ b y ~ a ~ p a t i e n t ~ s i m u l t a n e o u s l y ~ d u r i n g ~ t h e ~ i n d e x ~ p e r i o d . ~} \\
{ }^{d} \text { Total medication class is defined as the number of all therapy area drug categories used by a patient simultaneously during the index period. } \\
C D S=\text { chronic disease score; OOP = out-of-pocket; } S D=\text { standard deviation. }\end{array}$} \\
\hline
\end{tabular}

a commercial plan (mean range, 30\%-98\%). Stratum 1 had the lowest mean age (46.6 years) and highest CDS (7.0) and was the only group with patients covered by a Medicaid plan (28\%). Stratum 2 had the highest proportion of patients in a commercial plan (98\%). Stratum 3 had the highest mean household income $(\$ 77,028)$, lowest CDS (5.4), and second-highest proportion of patients in a commercial plan (94\%). Stratum 4 had the lowest mean household income $(\$ 62,893)$ and the highest proportion of patients in a Medicare Part D plan (60\%).

Similar to the index period, the covariates were balanced across the 2 OOP cost groups $(>\$ 35$ and $\leq \$ 35)$ for the diabetes OOP pharmacy cost analysis group, although significant differences were observed for some covariates. The mean patient age ranged from 52.3 to 56.1 years, mean CDS ranged from 6.0 to 6.5 , mean number of diabetes medication classes ranged from 1.5 to 3.2 , and mean household income ranged from $\$ 60,763$ to $\$ 79,373$ (Table 2B). Most patients were married (mean range, $76 \%-94 \%$ ) and used a commercial plan (mean range, 55\%-85\%). Stratum 1 had the lowest mean household income $(\$ 60,763)$ and was the only group with patients covered by a Medicaid plan (28\%). Stratum 2 reported the highest proportion of patients in a Medicare Part D plan (23\%). Stratum 3 had the second-highest number of diabetes medication classes (2.2) and secondhighest proportion of patients in a commercial plan (78\%). Stratum 4 had the highest mean household income $(\$ 79,373)$, highest total number of diabetes medication classes (3.2), and highest proportion of patients in a commercial plan (85\%).

\section{P Pharmacy Costs and Adherence}

In the unadjusted analysis for the overall population, antihyperglycemic medication adherence decreased as the diabetes OOP pharmacy costs increased. For patients on basal insulin, a continuous decrease in antihyperglycemic medication adherence was observed for diabetes OOP pharmacy cost levels $\geq \$ 75$, with patients in the diabetes OOP pharmacy cost levels \$75-\$99.99 reporting 464 days of antihyperglycemic medication coverage compared with 508 days in the lowest diabetes OOP pharmacy cost levels (\$0-\$9.99). Similarly, patients on 


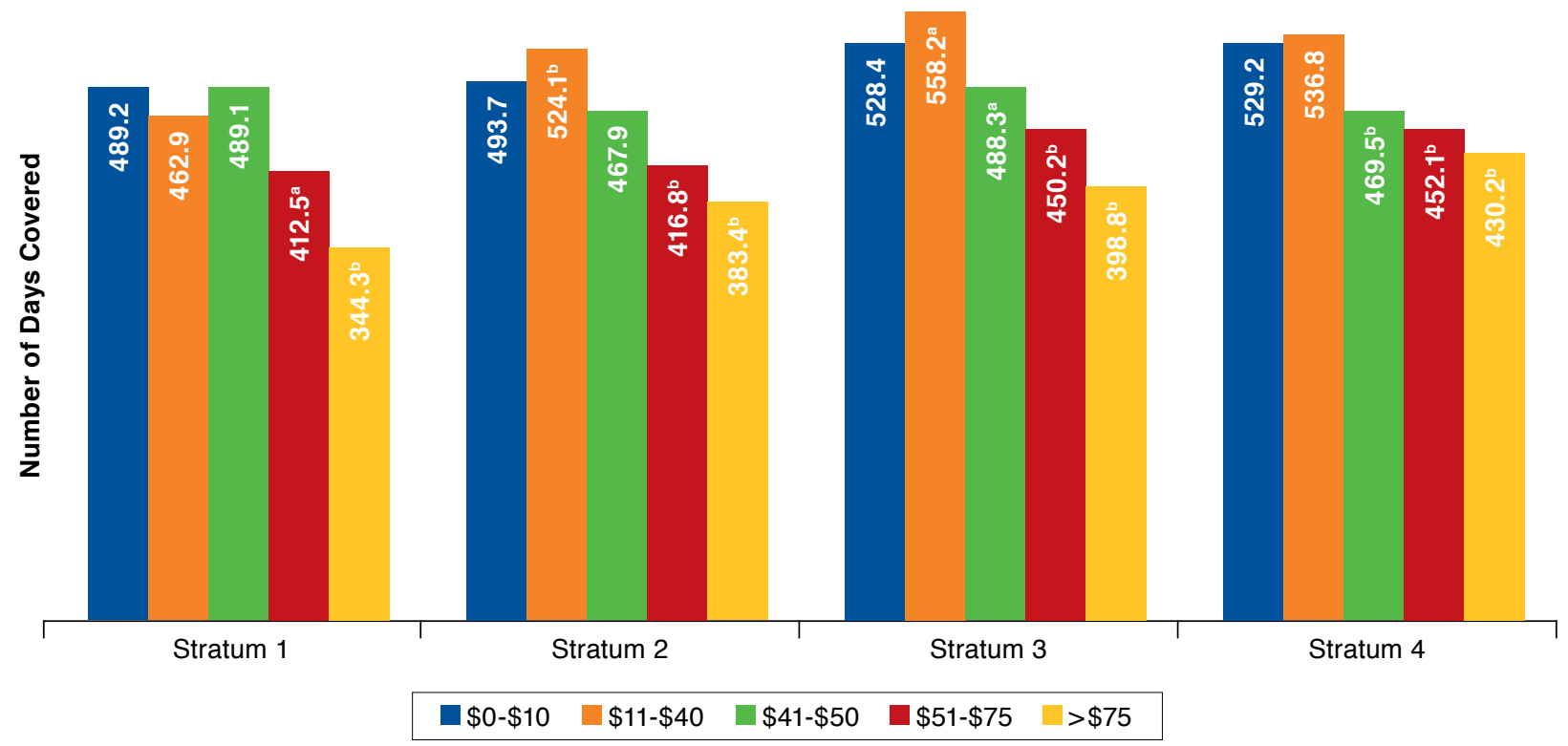

Note: Patients were divided into 4 strata based on Tukey's Five Number Summary to minimize the impact of confounding variables. P values indicate comparison with diabetes OOP pharmacy cost levels $\$ 0-\$ 10$ in respective strata.

aP $<0.05$.

${ }^{b} P<0.0001$.

OOP= out-of-pocket

rapid-acting insulin experienced a steady decrease in antihyperglycemic medication adherence for diabetes OOP pharmacy cost levels $\geq \$ 40$, with patients in the diabetes OOP pharmacy cost levels $\$ 40$ - $\$ 49.99$ reporting 401 days of coverage compared with 442 days in the lowest diabetes OOP pharmacy cost levels. A similar trend was observed for glucagon-like peptide-1 receptor agonists, with a steady reduction in adherence observed for diabetes OOP pharmacy cost levels \$30-\$39.99 (422 days of coverage) and above. For dipeptidyl peptidase-4 inhibitors, adherence decreased steadily up to diabetes OOP pharmacy cost levels $\$ 100-\$ 124.99$ (427 days of coverage) and a slight increase in adherence was observed for diabetes OOP pharmacy cost levels $\geq \$ 125$ (433 days of coverage).

The propensity-stratified multivariate regression model revealed an overall negative relationship between diabetes OOP pharmacy costs and antihyperglycemic medication adherence across several OOP cost levels. ${ }^{27}$ The likelihood of adherence was significantly decreased for patients with diabetes OOP pharmacy costs $>\$ 50(P<0.05)$ compared with those with the lowest diabetes OOP pharmacy cost levels $(\$ 0-\$ 10)$ in each of the 4 strata (Figure 2). Diabetes OOP pharmacy cost levels $\$ 51-\$ 75$ appeared to be the threshold at which significant reduction in adherence was observed across all strata, with patients experiencing 77-78 fewer days of coverage $(P<0.05)$ when compared with the lowest diabetes OOP pharmacy cost levels. The adherence levels reduced further for the higher diabetes OOP pharmacy cost levels, and patients with the highest diabetes OOP pharmacy costs $(>\$ 75)$ had 99-145 fewer days of coverage $(P<0.0001)$ compared with those with the lowest OOP cost levels.

Results from the sensitivity analysis revealed a negative association between total OOP pharmacy costs and antihyperglycemic medication adherence across several OOP cost levels (Figure 3). Adherence dropped significantly as OOP costs increased, especially for patients with total OOP pharmacy cost levels $>\$ 90(P<0.05)$ compared with those with the lowest OOP costs ( $\$ 0-\$ 30)$. Total OOP pharmacy cost levels $\$ 91-\$ 150$ appeared to be the threshold at which adherence reduced significantly across each stratum, and patients experienced 51-83 fewer days of coverage $(P<0.0001)$ when compared with the lowest total OOP pharmacy cost levels.

Similar findings were reported for index drug category OOP costs. Across each stratum, patients with higher index drug category OOP costs were less adherent to antihyperglycemic medication than patients with lower OOP costs. For strata 2 and 4 , the index drug category OOP cost levels $\$ 11-\$ 40$ appeared as the threshold at which antihyperglycemic medication adherence reduced significantly $(P<0.0001$; compared with OOP cost levels $\$ 0$-\$10); whereas the threshold 


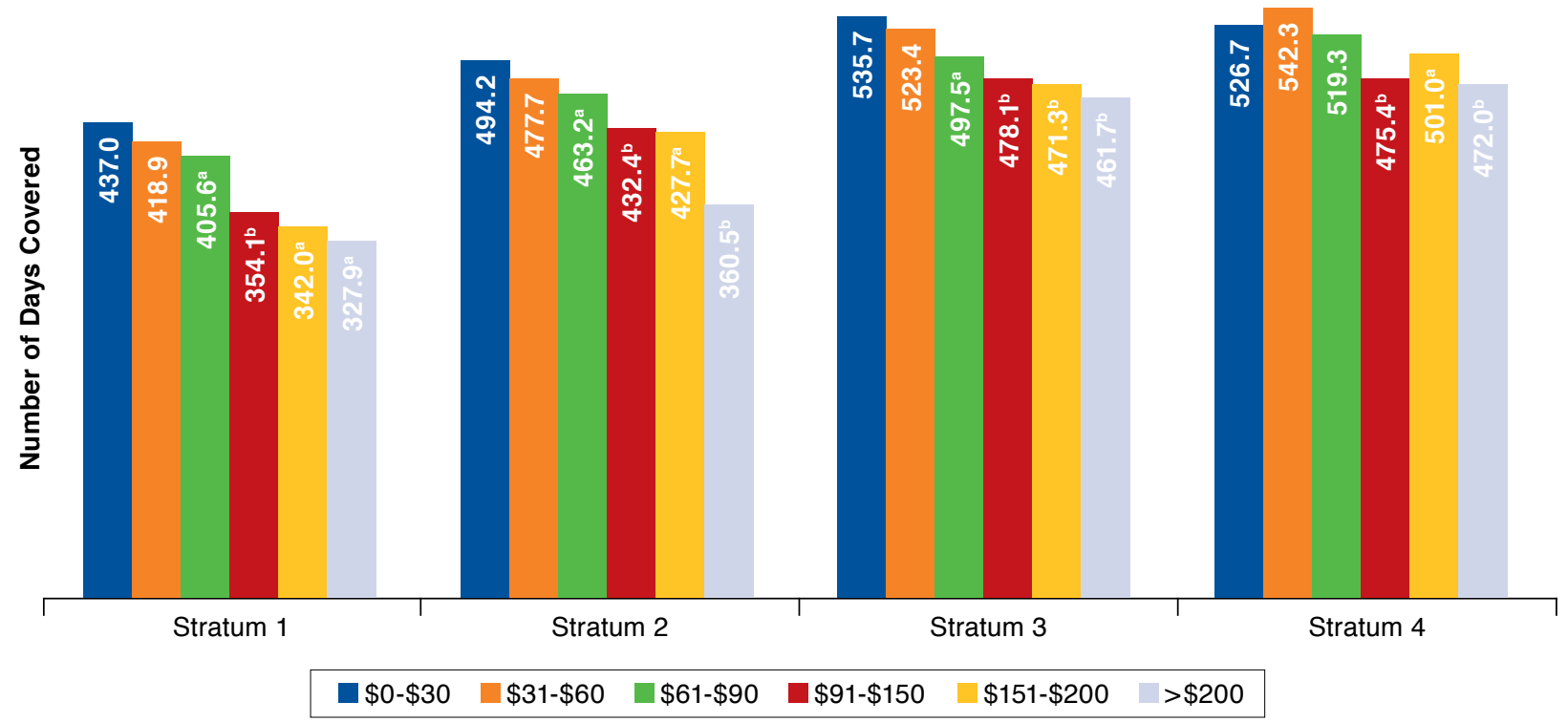

Note: Patients were divided into 4 strata based on Tukey's Five Number Summary to minimize the impact of confounding variables. P values indicate comparison with total OOP pharmacy cost levels $\$ 0$ - $\$ 10$ in respective strata.

$a_{P}<0.05$.

${ }^{b} P<0.0001$.

OOP=out-of-pocket

was $>\$ 75$ and $\$ 51-\$ 75$ for stratum 1 and stratum 3 , respectively $(P<0.05$, for each comparison).

\section{Discussion}

Adherence was consistently and negatively associated with OOP pharmacy costs in patients with T2DM. Within each stratification, the 3-year adherence decreased as OOP pharmacy costs increased. Similar findings have been observed in a literature review assessing the association of patient cost sharing and adherence, wherein $85 \%$ of articles reported that an increase in patients' medication cost sharing was significantly associated with a decrease in adherence. ${ }^{15}$

While not completely linear, adherence dropped with each level of OOP pharmacy cost increase, adjusting for age, income level, CDS, medication burden, and sociodemographic and clinical characteristics. The negative association of the level of OOP costs and adherence was also reported in earlier studies conducted in patients with T2DM as well as for other chronic conditions. ${ }^{19-21}$ A U.S. study demonstrated that an increase in cost sharing by $\$ 10$ resulted in a $6.2 \%$ decrease in antihyperglycemic medication adherence, and nonadherence led to an increase in diabetes-related complications and subsequent costs. ${ }^{20}$ Put into context, for every $\$ 10$ increase in copay, a patient on oral antihyperglycemic medication therapy was reported to be $26 \%$ more likely to become nonadherent. ${ }^{19}$
On the contrary, a reduction in copayment for antihyperglycemic medications from $\$ 15.3$ to $\$ 10.1$ was reported to increase the probability of adherence from $75.3 \%$ to $82.6 \% .^{21}$ These findings indicate that increases in the patients' share of copay by health care plans may indirectly lead to poor outcomes and increases in health care costs due to nonadherence.

It is apparent from this study that there is a threshold at which OOP pharmacy costs become a barrier to antihyperglycemic medication adherence. At index period, the threshold for index drug category OOP costs appeared to differ widely between the 4 strata. However, the threshold of OOP costs did not vary widely across strata when diabetes OOP pharmacy costs and total OOP pharmacy costs were considered. For the diabetes OOP pharmacy cost analysis, $\$ 51-\$ 75$ was identified as the threshold of OOP costs that led to significant reduction in adherence. Over the 3-year follow-up period, this reduction in adherence corresponded to a reduction in the proportion of days covered (PDC) ${ }^{28}$ from $44.7 \%$ to $48.3 \%$ in the lowest diabetes OOP pharmacy cost level to $37.7 \%$ to $41.3 \%$ in the $\$ 51-\$ 75$ cost level. The threshold appeared to be higher (\$91-\$150) for the total OOP pharmacy cost analysis, which could be due to the higher costs associated with all medications when compared with the use of diabetes medications only. 
An earlier study conducted between 2002 and 2006 observed a significant decrease in oral antihyperglycemic medication adherence with copay costs $>\$ 20$ when compared with copay costs of $<\$ 10 \quad(P<0.001){ }^{19}$ This study reports a higher threshold of OOP pharmacy costs that may be due to the increase in medication costs and overall health care expenditures over the years. Furthermore, recent studies conducted for other chronic diseases have reported a high threshold of OOP costs at which significant prescription abandonment is observed. ${ }^{29,30}$ In a study conducted on patients with rheumatoid arthritis, the likelihood of prescription abandonment was significantly increased for OOP costs $>\$ 250$ per prescription when compared with $\$ 0$ - $\$ 250$ OOP cost levels $(P<0.001){ }^{30}$ Another study observed significantly higher new therapy prescription abandonment rates with OOP costs $>\$ 100$ for tumor necrosis factor blockers $(P<0.05)$ and $>\$ 200$ for multiple sclerosis medications $(P<0.001)$, when compared with OOP costs $\leq \$ 100$, respectively. ${ }^{29}$

In the unadjusted analysis, a correlation between diabetes OOP pharmacy costs and antihyperglycemic medication adherence was observed among patients with T2DM using insulin, with continuous decrease in adherence observed for OOP cost levels $\geq \$ 75$ in basal insulin users and $\geq \$ 40$ in rapidacting insulin users, respectively. The difference in the OOP cost threshold could be due to the higher cost burden of rapidacting insulins compared with that of basal insulins. ${ }^{31}$

\section{Limitations}

This study has several limitations. First, the study population was drawn from a sample of insured patients from an observational health insurance claims database, and the findings may not be applicable to the general population.

Second, there is a limitation in using claims-based data for estimating adherence. The pharmacy claims database provides information on prescriptions filled and not prescriptions written or if the patients actually took medications. Thus, drug acquisition was measured rather than actual drug exposure data. However, the predictive validity of pharmacy dispensing records for measuring the cumulative medication exposure has been established. ${ }^{32}$ It should be noted that pharmacy claims data were also used to measure adherence to injectables. This method has its limitations, as it does not accurately capture the amount of medication contained in a single dose and does not take into account the impact of dosing frequency and dosing adjustments. ${ }^{33-35}$

Third, the potential association of nondocumented factors such as duration of diabetes, perceived ease of use of antihyperglycemic medication, and occurrence of adverse reactions could not be determined. Immortal time bias could potentially affect the study results, because patients who were very ill and died may have had higher OOP costs. However, considering the mean age of the patients included in the study, the impact of immortal time bias is not expected to be meaningful.

Fourth, the impact of the Medicare Part D coverage gap or the Medicare donut hole on OOP costs and adherence was not assessed in the study. Also, the introduction of the Patient Protection and Affordable Care Act and other changes in the health care system in the past 5 years may further limit the applicability of the findings.

Finally, as the study examined the impact of OOP pharmacy costs on adherence in patients with T2DM who newly initiated branded antihyperglycemic medication in 2011, the results may not be generalizable to patients with T2DM continuing treatment and those initiating generic antihyperglycemic medications.

Despite these limitations, this study has some important strengths. Although previous studies have assessed the impact of OOP costs on adherence, present-day data are required, considering the increasing trend of plans to shift costs to the patient. This study provides contemporary data on the impact of diabetes OOP pharmacy costs as well as total OOP pharmacy costs on antihyperglycemic medication adherence. The study findings indicate that adherence decreases with an increase in OOP costs irrespective of the category of OOP pharmacy costs or the confounding factors studied. Also, the study identifies a threshold at which diabetes and total OOP pharmacy costs lead to significant reduction in adherence in patients with T2DM.

These results may indicate that plan designs in which patients are responsible for paying a larger proportion of pharmacy expenses may be counterproductive to medication adherence. Findings from this study may be useful to inform the design of health care plans to achieve optimal adherence and improve long-term outcomes and disease management in patients with T2DM. However, future studies are needed to determine if savings from reduction in medication use due to increased patient cost sharing may be offset by the poor health outcomes and increased costs associated with lower medication adherence.

\section{Conclusions}

Overall, this study has shown that OOP pharmacy cost is negatively associated with antihyperglycemic medication adherence in patients with T2DM and that there is a threshold of diabetes OOP pharmacy costs (\$51-\$75) and total OOP pharmacy costs (\$91-\$150) at which a significant decrease in adherence occurs. Drug insurers and employers should be aware of the negative outcomes associated with nonadherence and should design health care plans to include modest copays that do not lead to nonadherence and a potential consequent increase in overall health care expenditures in patients with T2DM. 


\section{Authors}

WENDY S. BIBEAU, PhD, MEd, and HAODA FU, PhD, Eli Lilly and Company, Indianapolis, Indiana. APRIL D. TAYLOR, MSN, CNS, BC-ADM, and ANITA Y.M. KWAN, MSc, Lilly USA, Indianapolis, Indiana.

AUTHOR CORRESPONDENCE: Anita Y.M. Kwan, MSc, Lilly USA, Lilly Corporate Center, Indianapolis, IN 46285. Tel.: 317.655.1952; E-mail:kwanan@lilly.com.

\section{DISCLOSURES}

This study was funded by Eli Lilly and Company. Eli Lilly and Company was involved in the study design; collection, analysis, and interpretation of data; preparation of the manuscript; and decision to submit for publication. Fu is an employee of Eli Lilly and Company. Taylor and Kwan are employees of Lilly USA. Fu and Kwan hold stock or stock options in Eli Lilly and Company. Bibeau was an employee of Eli Lilly and Company at the time of this study and initial submission of this manuscript. Bibeau is currently employed by Janssen Scientific Affairs.

The abstract for this study was presented at the AMCP Managed Care \& Specialty Pharmacy Annual Meeting 2016; April 19-22, 2016; San Francisco, California.

Bibeau and Fu contributed to the study design and collected the data. All authors contributed equally to data interpretation and manuscript preparation and revision.

\section{ACKNOWLEDGMENTS}

The authors acknowledge IMS Health for its contribution to data collection and analysis, and Pixy Banerjee, MPharm, an employee of Eli Lilly, for medical writing assistance with this manuscript.

\section{REFERENCES}

1. Centers for Disease Control and Prevention. National diabetes statistics report, 2014: estimates of diabetes and its burden in the United States. 2014 U.S. Department of Health and Human Services. Atlanta, GA. Available at: https://www.cdc.gov/diabetes/pubs/statsreportl4/national-diabetes-reportweb.pdf. Accessed September 30, 2016.

2. Mathers CD, Loncar D. Projections of global mortality and burden of disease from 2002 to 2030. PLoS Med. 2006;3(11):e442.

3. American Diabetes Association. Standards of medical care in diabetes2016: summary of revisions. Diabetes Care. 2016;39(Suppl 1):S4-5. Available at: http://care.diabetesjournals.org/content/39/Supplement_1/S4. Accessed September 30, 2016.

4. Bailey CJ, Kodack M. Patient adherence to medication requirements for therapy of type 2 diabetes. Int J Clin Pract. 2011;65(3):314-22.

5. Garcia-Perez LE, Alvarez M, Dilla T, Gil-Guillen V, Orozco-Beltran D. Adherence to therapies in patients with type 2 diabetes. Diabetes Ther. 2013;4(2):175-94

6. Ho PM, Bryson CL, Rumsfeld JS. Medication adherence: its importance in cardiovascular outcomes. Circulation. 2009;119(23):3028-35.

7. Iuga AO, McGuire MJ. Adherence and health care costs. Risk Manag Healthc Policy. 2014;7:35-44.

8. Osterberg L, Blaschke T. Adherence to medication. N Engl J Med. 2005;353(5):487-97.

9. Briesacher BA, Andrade SE, Fouayzi H, Chan KA. Comparison of drug adherence rates among patients with seven different medical conditions. Pharmacotherapy. 2008;28(4):437-43.
10. Benjamin RM. Medication adherence: helping patients take their medicines as directed. Public Health Rep. 2012;127(1):2-3.

11. MacEwan JP, Sheehan J, Yin W, et al. Penny-wise, pound foolish: association between medication adherence, out-of-pocket expenses, and healthcare costs in Medicare patients with type 2 diabetes. Diabetes. 2015;64(Suppl 1): 262-OR

12. Jha AK, Aubert RE, Yao J, Teagarden JR, Epstein RS. Greater adherence to diabetes drugs is linked to less hospital use and could save nearly $\$ 5$ billion annually. Health Aff (Millwood). 2012;31(8):1836-46.

13. Roebuck MC, Liberman JN, Gemmill-Toyama M, Brennan TA. Medication adherence leads to lower health care use and costs despite increased drug spending. Health Aff (Millwood). 2011;30(1):91-99.

14. Centers for Medicare $\&$ Medicaid Services. NHE fact sheet. Historical NHE, 2014. Available at: https://www.cms.gov/research-statistics-data-andsystems/statistics-trends-and-reports/nationalhealthexpenddata/nhe-factsheet.html. Accessed September 30, 2016

15. Eaddy MT, Cook CL, O'Day K, Burch SP, Cantrell CR. How patient cost-sharing trends affect adherence and outcomes: a literature review. P T. 2012;37(1):45-55

16. America's Health Insurance Plans, Center for Policy and Research. 2015 census of health savings account-high deductible health plans. Available at: https://ahip.org/wp-content/uploads/2015/11/HSA_Report.pdf. Accessed September 30, 2016.

17. Braithwaite S, Shirkhorshidian I, Jones K, Johnsrud M. The role of medication adherence in the U.S. healthcare system. June 2013. Available at: http://static.correofarmaceutico.com/docs/2013/06/24/adher.pdf. Accessed September 30, 2016.

18. Reiss SK, Ross-Degnan D, Zhang F, Soumerai SB, Zaslavsky AM, Wharam JF. Effect of switching to a high-deductible health plan on use of chronic medications. Health Serv Res. 2011;46(5):1382-401.

19. Barron J, Wahl P, Fisher M, Plauschinat C. Effect of prescription copayments on adherence and treatment failure with oral antidiabetic medications. P T. 2008;33(9):532-53.

20. Gibson TB, Song X, Alemayehu B, et al. Cost sharing, adherence, and health outcomes in patients with diabetes. Am J Manag Care. 2010;16(8):589-600.

21. Zeng F, An JJ, Scully R, Barrington C, Patel BV, Nichol MB. The impact of value-based benefit design on adherence to diabetes medications: a propensity score-weighted difference in difference evaluation. Value Health. 2010;13(6):846-52.

22. Viboud C, Charu V, Olson D, et al. Demonstrating the use of high-volume electronic medical claims data to monitor local and regional influenza activity in the US. PLoS One. 2014;9(7):e102429.

23. Von Korff M, Wagner EH, Saunders K. A chronic disease score from automated pharmacy data. J Clin Epidemiol. 1992;45(2):197-203.

24. Chang HY, Weiner JP, Richards TM, Bleich SN, Segal JB. Validating the adapted Diabetes Complications Severity Index in claims data. Am J Manag Care. 2012;18(11):721-26.

25. IMS Institute for Healthcare Informatics. Patient savings program use analysis. February 2014. Available at: https://www.imshealth.com/files/web/ IMSH\%20Institute/Healthcare\%20Briefs/Patient_Savings_Program_Impact_ Analysis.pdf. Accessed September 30, 2016.

26. Adisa R, Fakeye TO. Effect of number and type of antidiabetes medications on adherence and glycemia of ambulatory type 2 diabetes patients in southwestern Nigeria. Pharm Pract (Granada). 2013;11(3):156-65.

27. Bibeau WS, Fu H. Impact of out-of-pocket costs on branded medication adherence and outcomes among patients with type 2 diabetes. J Manag Care Spec Pharm. 2016;22(Suppl 4a):Abstract E21. Available at: http://www.jmcp. org/doi/abs/10.18553/jmcp.2016.22.4.S1.

28. Benner JS, Glynn RJ, Mogun H, Neumann PJ, Weinstein MC, Avorn J. Long-term persistence in use of statin therapy in elderly patients. JAMA. 2002;288(4):455-61. 
29. Gleason PP, Starner CI, Gunderson BW, Schafer JA, Sarran HS. Association of prescription abandonment with cost share for high-cost specialty pharmacy medications. J Manag Care Pharm. 2009;15(8):648-58. Available at: http://www.jmcp.org/doi/10.18553/jmcp.2009.15.8.648.

30. Hopson S, Saverno K, Liu LZ, et al. Impact of out-of-pocket costs on prescription fills among new initiators of biologic therapies for rheumatoid arthritis. J Manag Care Spec Pharm. 2016;22(2):122-30. Available at: http:// www.jmcp.org/doi/10.18553/jmcp.2016.14261.

31. Grunberger G. Insulin analogs-are they worth it? Yes! Diabetes Care. 2014;37:1767-70

32. Choo PW, Rand CS, Inui TS, et al. Validation of patient reports, automated pharmacy records, and pill counts with electronic monitoring of adherence to antihypertensive therapy. Med Care. 1999;37(9):846-57.
33. Campagna EJ, Muser E, Parks J, Morrato EH. Methodological considerations in estimating adherence and persistence for a long-acting injectable medication. J Manag Care Spec Pharm. 2014;20(7):756-66. Available at: http://www.jmcp.org/doi/10.18553/jmcp.2014.20.7.756.

34. Slabaugh SL, Bouchard JR, Li Y, Baltz JC, Meah YA, Moretz DC. Characteristics relating to adherence and persistence to basal insulin regimens among elderly insulin-naive patients with type 2 diabetes: pre-filled pens versus vials/syringes. Adv Ther. 2015;32(12):1206-21.

35. Fairman KA, Motheral B. Evaluating medication adherence: which measure is right for your program? J Manag Care Pharm. 2000;6(6):499-506. Available at: http://www.jmcp.org/doi/abs/10.18553/jmcp.2000.6.6.499. 
APPENDIX A USC Codes for Index Drug Categories

\begin{tabular}{|c|c|c|c|}
\hline \multirow{4}{*}{$\begin{array}{l}\text { Drug Categorya } \\
\text { DPP-4 inhibitors }\end{array}$} & \multirow{4}{*}{$\frac{\text { USC Category }}{39261 / 39262}$} & \multicolumn{2}{|l|}{ Brand Name } \\
\hline & & - Janumet/Janumet XR & - Kombiglyze XR \\
\hline & & - Januvia & - Onglyza \\
\hline & & - Juvisync & - Tradjenta \\
\hline \multirow[t]{2}{*}{ GLP-1 receptor agonists } & \multirow[t]{2}{*}{39251} & - Bydureon & - Victoza \\
\hline & & - Byetta & \\
\hline \multirow{2}{*}{ Basal insulin } & \multirow[t]{2}{*}{39133} & - Lantus/Lantus SoloSTAR & - Insulatard NPH Human \\
\hline & & - Levemir/Levemir FlexPen/Levemir FlexTouch & \\
\hline \multirow[t]{5}{*}{ Rapid-acting insulin } & \multirow[t]{5}{*}{39131} & - Apidra/Apidra SoloSTAR & - Novolin \\
\hline & & - Humalog/Humalog KwikPen & - Novolog \\
\hline & & - Humalog Mix & - Relion \\
\hline & & - Humalin & - Velosulin \\
\hline & & - Mixtard & \\
\hline
\end{tabular}

a Combination formulations of medications within these categories have been rolled up under the primary active component, e.g., Janumet is rolled up under Januvia within the DPP-4 inhibitor category.

DPP-4 = dipeptidyl peptidase-4; GLP-1 = glucagon-like peptide-1; USC= Uniform System of Classification.

\section{APPENDIX B Targeted Comorbidities of Interest}

\begin{tabular}{|c|c|c|c|}
\hline Disease & ICD-9-CM Diagnosis Codes & $\begin{array}{l}\text { ICD-9-CM Procedure } \\
\text { Codes }\end{array}$ & CPT Codes \\
\hline T1DM & $250 . x 1$ or $250 . x 3$ & - & - \\
\hline T2DM & $250 . x 0$ or $250 . x 2$ & - & - \\
\hline NAFLD/NASH & $571.8 \mathrm{x}$ & - & - \\
\hline Hypertension & 401.xx, 402.xx, 403.xx, 404.xx, 405.xx & - & - \\
\hline Dyslipidemia & 272.xx & - & - \\
\hline Obesity & $278.00,278.01, \mathrm{~V} 85.3 \mathrm{x}, \mathrm{V} 85.4 \mathrm{x}$ & - & - \\
\hline Bariatric surgery & V45.86, 44.31x, 44.38x, 44.39x, 44.68x, 44.95x & - & $43644,43645,43770,43775,43842-43848$ \\
\hline Gastrointestinal side effects & $\begin{array}{l}536.8 x, 536.3 x, 536.4 x, 787.01,780.02,787.03 \\
787.3,789.0 x, 564.00,564.01,564.02,564.03 \\
564.09,787.91\end{array}$ & - & - \\
\hline Amputations and ulcerations & 997.6x, V49.7x, V49.6x & $84.1 x$ & $28800-28825,27880-27889$ \\
\hline Mental illness & 290.xx-311.xx & - & - \\
\hline Anxiety & $300 . x x$ & - & - \\
\hline Depression & 296.2x, 296.3x, 300.4x, 309.0x, 309.1x, 311.xx & - & - \\
\hline
\end{tabular}

Note: One occurrence of the codes was required to capture the targeted comorbidities of interest.

$C P T=$ Current Procedural Terminology; ICD-9-CM = International Classification of Diseases, Ninth Revision, Clinical Modification; NAFLD/NASH =nonalcoholic fatty liver disease/nonalcoholic steatohepatitis; TIDM = type 1 diabetes mellitus; T2DM=type 2 diabetes mellitus. 